\title{
Video Article \\ Cheek Injection Model for Simultaneous Measurement of Pain and Itch-related Behaviors
}

\author{
Yu Yamanoi ${ }^{1,2,3,4}$, Hiroki Kittaka ${ }^{1,2,5}$, Makoto Tominaga ${ }^{1,2,3,6}$ \\ ${ }^{1}$ Thermal Biology Group, Exploratory Research Center on Life and Living Systems (ExCELLS) \\ ${ }^{2}$ Division of Cell Signaling, National Institute for Physiological Sciences \\ ${ }^{3}$ Department of Physiological Sciences, Graduate University for Advanced Studies (SOKENDAI) \\ ${ }^{4}$ Research Laboratory, Ikedamohando Co., Ltd. \\ ${ }^{5}$ Department of Molecular Biomedical Sciences, College of Veterinary Medicine, North Carolina State University \\ ${ }^{6}$ Institute for Environmental and Gender-Specific Medicine, Juntendo University
}

Correspondence to: Makoto Tominaga at tominaga@nips.ac.jp

URL: https://www.jove.com/video/58943

DOI: doi:10.3791/58943

Keywords: Neuroscience, Issue 151, scratching, itch, pain, behavior, histamine, capsaicin

Date Published: 9/27/2019

Citation: Yamanoi, Y., Kittaka, H., Tominaga, M. Cheek Injection Model for Simultaneous Measurement of Pain and Itch-related Behaviors. J. Vis. Exp. (151), e58943, doi:10.3791/58943 (2019).

\section{Abstract}

Itch was defined as "an unpleasant cutaneous sensation that provokes a desire to scratch" by Rothman in 1941. In mouse models, scratch bouts are typically counted to evaluate itch induced by pruritogens. However, previous reports have shown that algesic substances also induce scratching behaviors in a mouse neck injection model, which is the most common test used for scratching behaviors. This finding makes it difficult to study itch in mice. In contrast, capsaicin, a common algogen, reduced scratching behaviors in some neck injection experiments. Therefore, the effect of pain on scratching behaviors remains unclear. It is thus necessary to develop a method to concurrently investigate itch and pain sensation using behavioral tests. Here, a cheek injection model is introduced which can be used to simultaneously measure painand itch-related behaviors. In this model, pruritogens induce scratching behaviors while algesic substances induce wiping behaviors. Using this model, lysophosphatidic acid (LPA), an itch mediator found in cholestatic patients with itch, is shown to exclusively induce itch but not pain. However, in mouse models, LPA has been reported to be both a pruritogen and an algogen. Investigation into the effects of LPA in a mouse cheek injection model showed that LPA only induced scratching, but not wiping behaviors. This indicates that LPA acts as a pruritogen similarly in mice and humans, and demonstrates the utility of a cheek injection model for itch research.

\section{Video Link}

The video component of this article can be found at https://www.jove.com/video/58943/

\section{Introduction}

Itch was originally characterized as a sensation which induces scratching behaviors to remove harmful materials from the skin surface. However, itch has been the focus of therapies for unpleasant sensations caused by many diseases such as atopic dermatitis, neurogenic lesions, and cholestasis ${ }^{1}$. In these cases, itch is a serious unpleasant sensation similar to pain. Therefore, itch represents an important research target. Scratching behavior is the primary indicator of itch in animal experiments, and scratching behaviors can be induced in mice by injecting pruritogens into the skin on their neck ${ }^{2,3}$. However, a previous study showed that capsaicin, an algogen, also induces scratching behaviors in a neck injection model ${ }^{4}$, making it difficult to discriminate itch from pain in this model. In contrast, it has been found that capsaicin reduced scratching behaviors in a neck injection model, indicating that the effect of pain on scratching behaviors is complicated and depends on the experimental conditions. Therefore, simultaneous measurements of pain and itch-related behaviors would enable the precise analysis of pruritogens and algogens. Steven G. Shimada and Robert $\mathrm{H}$. LaMotte succeeded in simultaneous measurements of pain and itch-related behaviors by changing the injection site from the neck to the cheek ${ }^{4}$. In the cheek injection model, capsaicin induced wiping, but not scratching behaviors, while histamine, a pruritogen, only induced scratching behaviors. Therefore, this model enables the independent assessment of itch and pain, making cheek injection a useful model for itch research. When trying to determine if a test substance is a pruritogen and/or an algogen, this model provides more information than the neck injection model. This article describes a practical method for performing cheek injection and counting the bouts of scratching or wiping, and shows the utility of this method for evaluating scratching behaviors induced by lysophosphatidic acid (LPA).

\section{Protocol}

All procedures involving the care and use of animals were approved by the institutional Animal Care and Use Committee of the National Institute of Natural Sciences (16A074), and carried out in accordance with the guidelines of the National Institute for Physiological Sciences. 
NOTE: The original protocol was reported by Steven G. Shimada and Robert H. LaMotte ${ }^{4}$, and the present report describes these methods with several modifications, including the addition of a screen to prevent the animals from seeing each other, the absence of a mirror, the number of mice per recording, mouse strain, cage size, and recording time.

\section{Cheek Injection Model}

\section{Experiment preparation}

1. Cage

1. Set four cages under a camera as shown in Figure 1. Set a screen to prevent the mice from seeing each other. Put in each mouse to each cage and set an acrylic lid to prevent escape of mice. Note that recordings should be performed in an environment in which temperature, humidity and sound are controlled.

NOTE: Behaviors can also be recorded without a lid if the cage is sufficiently high.

2. Needle and syringe

1. Use a $29 \mathrm{G}$ or $30 \mathrm{G}$ needle attached to a $0.5 \mathrm{~mL}$ syringe for the injection of test substances. If the test substance solution is highly viscous, larger needles are required. However, please note that large needles pose additional physical side effects. NOTE: A needle connected to a syringe with a polyethylene tube can be used.

3. Video camera

1. Use a video camera having sufficiently large frame rates (30-60 frames/s) with relatively high resolution for the measurement of scratch bouts and wiping behaviors.

4. Software for video analysis

1. Use a video editing software for the video playback and analysis. Note that a fast-forward mode and a frame-by-frame playback mode are needed for precise analysis.

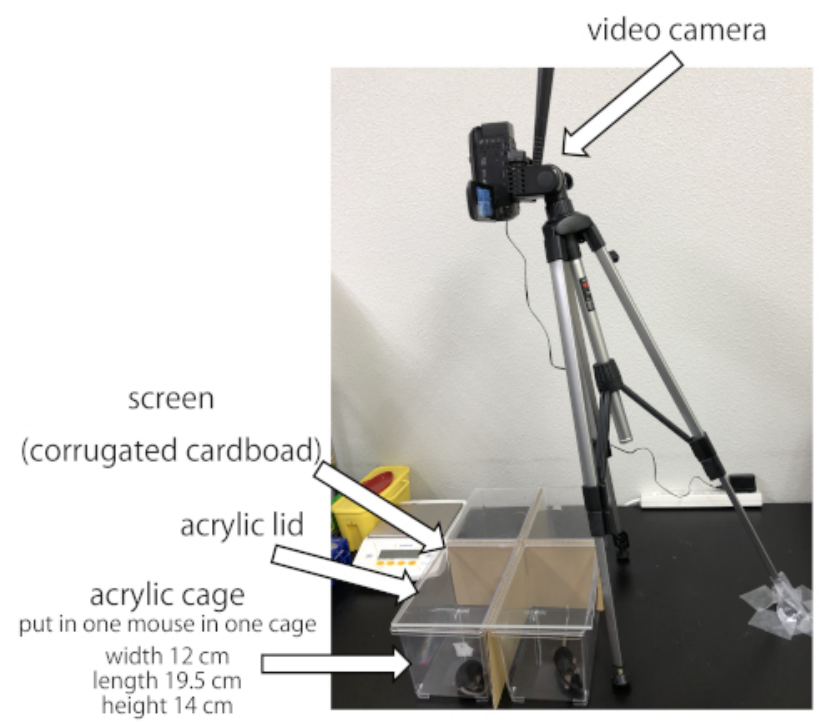

Figure 1: Video camera and cage set-up. Four acrylic cages with acrylic lids, for preventing escape of the mice, and a screen for preventing contagious itch are used. Please click here to view a larger version of this figure.

2. Injection and recording

1. One day prior to the experiment, shave the fur on the mouse's cheek with hair clippers (Movie 1). Before shaving, do not restrain the mice.

NOTE: CD-1 or C57BL/6 mice were mostly used in this model ${ }^{4,5}$. In this article, male 6-12 week-old C57BL/6N mice were used. Note that sensitivity to the chemicals could be different between the various mouse strains ${ }^{3,6,7}$.

2. One day after shaving, move the mouse to the recording cage for $1 \mathrm{~h}$ to acclimatize it to the recording conditions. If a topical application is used, apply it during this acclimation period. NOTE: In this report, $10 \mu \mathrm{L}$ of $2 \%(\mathrm{w} / \mathrm{v})$ diphenhydramine hydrochloride salt in saline was topically applied 30 min prior to recording.

3. Set a video camera above the cage, and start the video recording before the test substance injection. Inject a $10 \mu \mathrm{L}$ solution of the test substance intradermally into the shaved cheek under awake conditions (Movie 2). After the injection, return the mouse to the recording cage.

NOTE: In this report, $50 \mu \mathrm{g} / 10 \mu \mathrm{L}$ histamine, $40 \mu \mathrm{g} / 10 \mu \mathrm{L}$ capsaicin or vehicle ( $7 \%$ tween 80 in saline) were used as test substances.

4. Close the lid of the cage and continue video recording for $30 \mathrm{~min}$.

NOTE: The total recording time will depend on the experimental conditions.

\section{Behavioral analysis}


1. Count scratch bouts and wiping behaviors, respectively. Mice raise a hind leg towards the cheek and scratch several times over one or a few seconds, then put their leg down. This series of actions is counted as one scratch bout. Scratching of other areas should not be counted. Mice use a single foreleg for wiping and both forelegs for grooming. These actions should be distinguished. Only wiping behaviors are counted as pain-related behaviors.

NOTE: Each action is shown in Figure 2, Movie 3, Movie 4, and Movie 5.

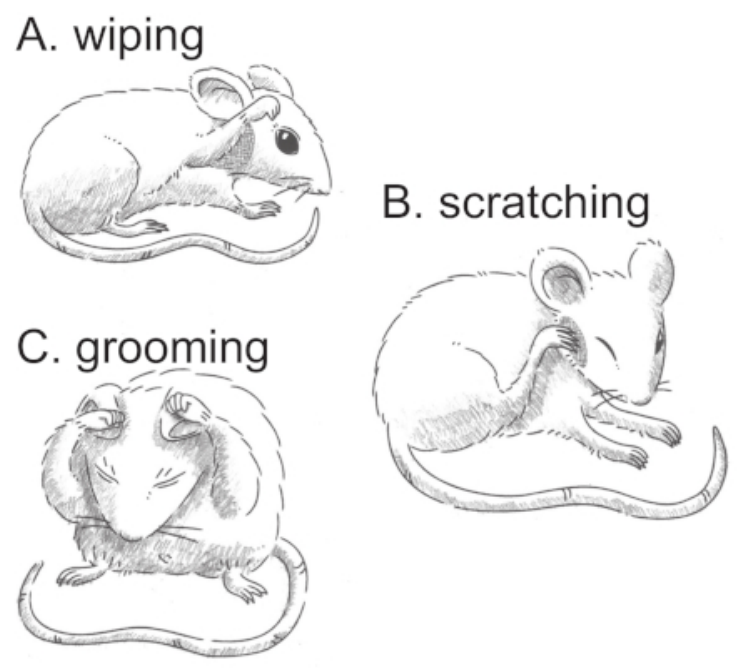

Figure 2: Typical mouse activity during recording. Panels $A-C$ are illustrations representing wiping behavior with the right foreleg $(\mathbf{A})$, scratching behavior with the right hind leg (B) and grooming behavior with both forelegs (C). Mice raise a hind leg towards the cheek and scratch several times over one or a few seconds, then put that leg down. This series of actions is counted as one scratch bout. Scratching of other areas should not be counted. Mice use a single foreleg for wiping and both forelegs for grooming. These actions should be distinguished. Only wiping behaviors are counted as pain-related behaviors. Please click here to view a larger version of this figure.

\section{Neck Injection Model}

1. Injection and recording

1. One day prior to the experiment, shave the fur on the nape of the mouse's neck with hair clippers after approximately $5 \%$ isoflurane inhalation for 2-3 min.

2. One day after shaving, move the mouse to the recording cage for $1 \mathrm{~h}$ to acclimatize it to the recording conditions.

3. Set a video camera above the cage (Figure 1), and start the video recording before the test substance injection. Inject a $10 \mu \mathrm{L}$ solution of the test substance intradermally into the shaved neck under awake conditions. After the injection, return the mouse to the recording cage.

NOTE: In this article, $50 \mu \mathrm{g} / 10 \mu \mathrm{L}$ histamine, $10 \mu \mathrm{g} / 10 \mu \mathrm{L}$ or $40 \mu \mathrm{g} / 10 \mu \mathrm{L}$ capsaicin, or vehicle (7\% tween 80 in saline) were used as test substances.

4. Close the lid of the cage and continue video recording for $30 \mathrm{~min}$.

NOTE: Total recording time will depend on the experimental conditions.

\section{Behavioral analysis}

1. Count scratch bout behaviors. Mice raise a hind leg towards the neck and scratch several times over one or a few seconds, then put that leg down. This series of actions is counted as one scratch bout. Scratching of other areas should not be counted.

NOTE: The action of scratching is similar to that in the cheek injection model, except that the location of the scratching is shifted to the neck.

\section{Representative Results}

In the neck injection model, histamine induced strong scratching behaviors (Figure 3 ). Vehicle (7\% tween 80 in saline) also induced scratching behaviors. A previous report showed that detergent induced itch by histamine production from keratinocytes ${ }^{8}$. Tween 80 could induce itch by similar mechanisms, whereas capsaicin reduced vehicle-induced scratching behaviors, contrary to a previous report ${ }^{4}$. It is possible that mouse strain differences could account for these different results. These findings indicate that the effect of pain on scratching behaviors is complicated Therefore, the simultaneous measurement of pain and itch-related behaviors may enable the precise analysis of pruritogens and algogens. In the cheek injection model, capsaicin did not induce scratching behaviors and only induced wiping behaviors, which are thought to be related to pain $^{9,10,11}$ (Figure 4). Capsaicin injection also increased grooming behaviors (data not shown). Following injection into the cheek, histamine mainly induced scratching behaviors with weak wiping behaviors. The application of an anti-histamine reagent, diphenhydramine, inhibited scratching. This result suggests that histamine-induced scratching behaviors are caused by the action of histamine on histamine receptors. Here, as an example, the effects of LPA in the cheek injection model are shown (Figure 5). LPA is an itch mediator found in cholestatic patients with 
itch. However, in mouse models, LPA was reported as both a pruritogen and an algogen ${ }^{12,13,14,15}$. Therefore, the effects of LPA were investigated in a cheek injection model and results showed that LPA induced scratching, but not wiping behaviors. This indicates that LPA is a pruritogen, but not an algogen, in mice similar to its effects in humans.

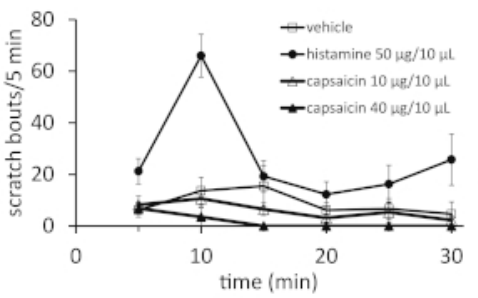

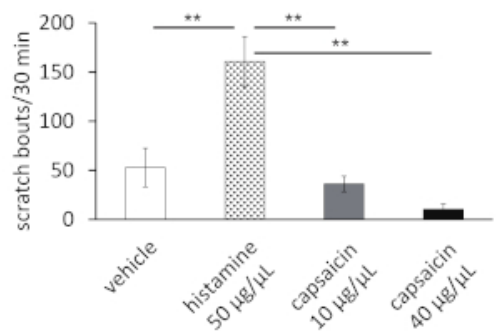

Figure 3: Effects of histamine and capsaicin in the neck injection model. (A) Scratching behaviors induced by the injection of vehicle (7\% tween 80 in saline, open square), histamine $(50 \mu \mathrm{g} / 10 \mu \mathrm{L}$ per site, filled circles) or capsaicin $(10 \mu \mathrm{g} / 10 \mu \mathrm{L}$ per site, open triangle; $40 \mu \mathrm{g} / 10 \mu \mathrm{L}$ per site, filled triangle) into the neck were assessed every $5 \mathrm{~min}$ for a total of $30 \mathrm{~min}$. (B) Quantitative analysis of the scratching behaviors during the 30 min period in panel A. \#\#P<0.01 by ANOVA followed by Bonferroni correction. Data are shown as the mean \pm SEM $(n=6-8)$. Please click here to view a larger version of this figure.
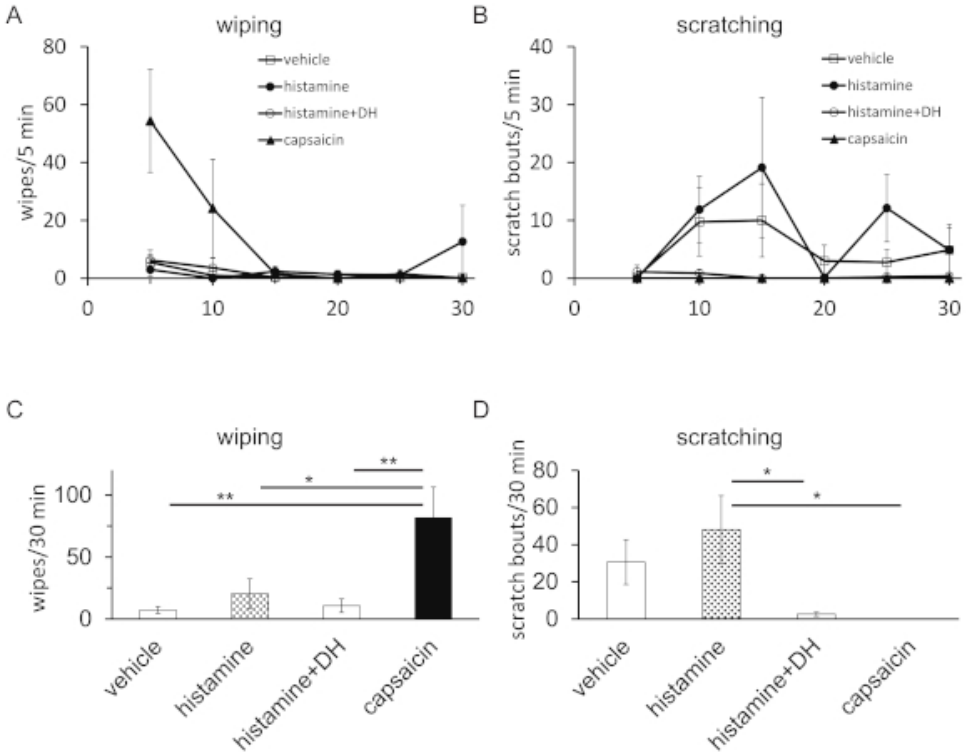

Figure 4: Effects of a pruritogen and an algogen in the cheek injection model. Wiping (A) and scratching (B) behaviors induced by injection of vehicle ( $7 \%$ tween 80 in saline, open square), histamine $(50 \mu \mathrm{g} / 10 \mu \mathrm{L}$ per site, filled circles) or capsaicin $(40 \mu \mathrm{g} / 10 \mu \mathrm{L}$ per site, filled triangle) into the right cheek of mice were assessed every $5 \mathrm{~min}$ for a total of $30 \mathrm{~min}$. The group shown as open circles was treated with $10 \mu \mathrm{L}$ of $2 \%$ (w/v) diphenhydramine hydrochloride salt (DHHCL) percutaneously 30 min prior to histamine injection $(50 \mu \mathrm{g} / 10 \mu \mathrm{L}$ per site). DHHCl was dissolved in saline. Quantitative analysis of the wiping (C) and scratching (D) behaviors during the 30 min period in panels $A$ and $B ; n=8$ animals per group. $\# P<0.05, \# \#<0.01$ by ANOVA followed by Bonferroni correction. Data are shown as the mean $\pm \mathrm{SEM}$. Please click here to view a larger version of this figure. 
A

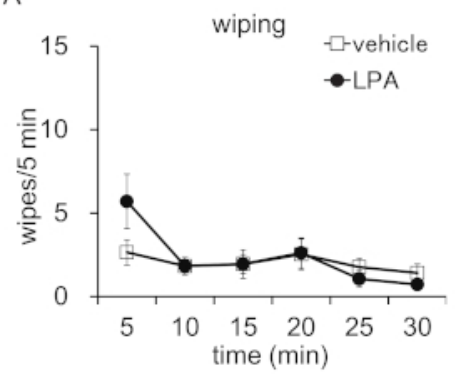

C

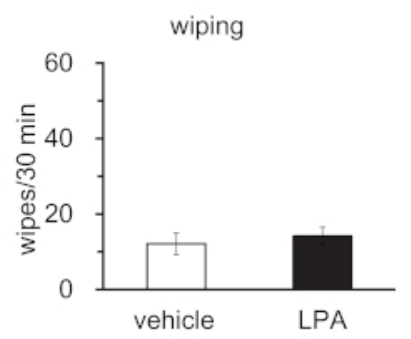

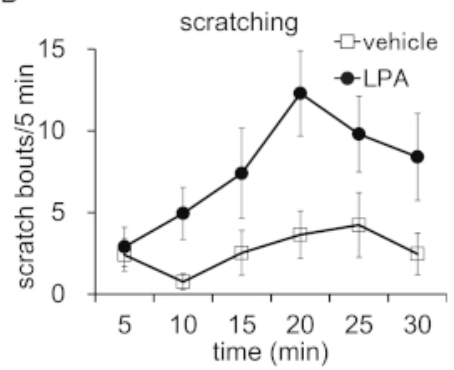

D

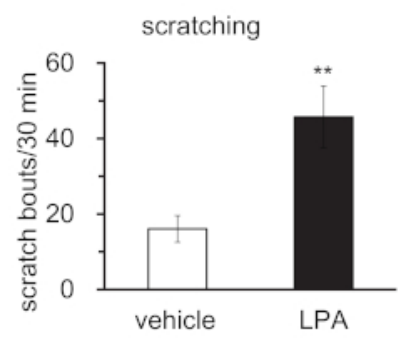

Figure 5: LPA induces scratching, but not wiping behaviors in the cheek injection model. Scratching (A) and wiping (B) behaviors induced by injection of vehicle (saline, open squares) or LPA $(100 \mathrm{nmol} / 10 \mu \mathrm{L}$ per site, filled circles) into the right cheek of mice were assessed every 5 min for a total of $30 \mathrm{~min}$. Quantitative analysis of the scratching $(\mathbf{C})$ and wiping (D) behaviors during the 30 min period in panels $A$ and $B ; n=17$ for vehicle and $\mathrm{n}=20$ for LPA. \#P<0.05, \#\#P<0.01 by Welch's $t$ test. Data are shown as the mean \pm SEM. This figure has been modified from Kittaka et al. ${ }^{5}$. Please click here to view a larger version of this figure.

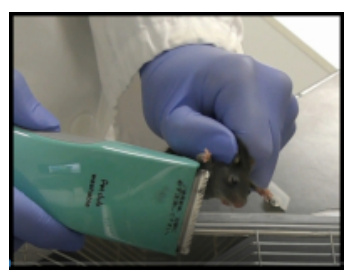

Movie 1: Movie demonstrating how to shave a mouse cheek. Please click here to view this video. (Right-click to download.)

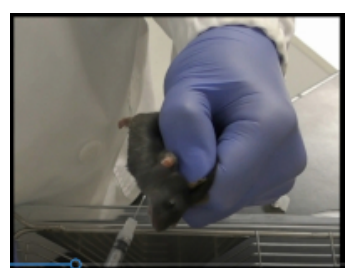

Movie 2: Movie demonstrating the injection into a mouse cheek. Please click here to view this video. (Right-click to download.)

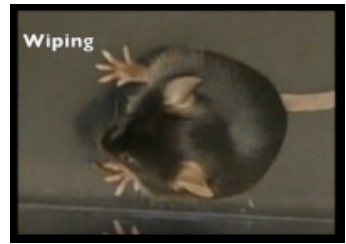

Movie 3: Movie demonstrating typical wiping behavior. This movie is shown at $25 \%$ speed. Please click here to view this video. (Right-click to download.) 


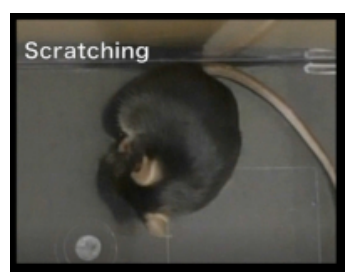

Movie 4: Movie of typical scratching behavior. Please click here to view this video. (Right-click to download.)

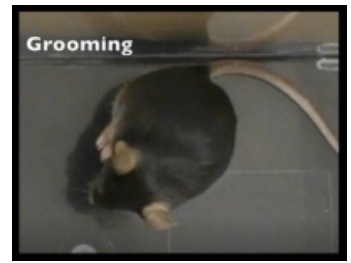

Movie 5: Movie of typical grooming behavior. This movie is shown at $25 \%$ speed. Please click here to view this video. (Right-click to download.)

\section{Discussion}

In this article, a cheek injection model is introduced and shown to be useful for the analysis of pain and itch-related behaviors.

A previous report showed that algesic substances induce scratching behaviors in a mouse neck injection model ${ }^{4}$. In contrast, capsaicin reduced scratch behaviors in other neck injection experiments (Figure 3). Therefore, the effect of pain on scratching behavior remains unclear. It is thus necessary to develop a method to concurrently investigate pain and itch through behavioral tests. By using a cheek injection model, the effect of a substance on both pain and itch can be evaluated.

It has previously been reported that intracellular LPA directly activates TRPV1 and TRPA1 as a mechanism of LPA-induced itch ${ }^{5}$. Accordingly, LPA-induced itch could be histamine independent. A previous study also showed that a cheek injection model can be used for the evaluation of histamine-independent itch ${ }^{16}$. Therefore, this model can be used for both histamine-dependent and -independent itch.

One of the critical steps in this experiment is the injection. Because this is an acute behavioral test, anesthesia cannot be used in the injection step as it might affect these behaviors. Therefore, the mouse needs to be held securely in order to perform a precise injection. When the hold on the animal is not secure, especially when injecting strong algogens, the mice may struggle, leading to an inaccurate injection volume and depth, followed by difficulty in interpreting the results. When handling a mouse, pull the skin on the nape between the thumb and forefinger to inhibit movement of the mouse.

Another critical step is the behavioral analysis. Because scratching is very rapid, the video needs to be watched carefully. Moreover, wiping can be difficult to distinguish from grooming. Therefore, the use of positive control chemicals such as capsaicin is recommended during the first experiment. Recording and replay conditions can also affect counting of both behaviors. If it is difficult to clearly observe the behaviors, mirrors can be used around the cages to enhance the viewing angles ${ }^{4}$.

It should be noted that the cheek injection model may show reduced quantitative accuracy for the tested chemicals than in the neck injection model. As shown in Figure 3 and Figure 4, the same amount of histamine induced more scratching behaviors in the neck model than in the cheek model, and showed smaller variation in the neck injection model. Once a tested substance has been shown to be a pruritogen with low algesic effects with the cheek injection model, the neck injection model is recommended for additional quantitative analysis. Even with these limitations, the cheek injection model is useful for the precise evaluation of test substances, especially in the field of itch research.

\section{Disclosures}

The authors have nothing to disclose.

\section{Acknowledgments}

We are grateful to Drs. Steven G. Shimada and Robert H. LaMotte (Yale University School of Medicine, Connecticut) from whose methods (reference 4) this technique was adapted. We also thank Mr. Kentaro Miyahara for creating the mouse illustrations. This study was supported by a Grant-in-Aid for Scientific Research from the Ministry of Education, Culture, Sports, Science and Technology in Japan (Nos. 15H02501 and $15 \mathrm{H} 05928$ to M.T., and No. 16K21691 to H.K.) and Uehara Memorial Foundation (to H.K.). We appreciate the introduction of the cheek injection method by Prof. LaMotte in Yale University.

\section{References}

1. Ikoma, A., Steinhoff, M., Stander, S., Yosipovitch, G., Schmelz, M. The neurobiology of itch. Nature Reviews Neuroscience. 7 (7), $535-547$, (2006). 
2. Kuraishi, Y., Nagasawa, T., Hayashi, K., Satoh, M. Scratching behavior induced by pruritogenic but not algesiogenic agents in mice. European Journal of Pharmacology. 275 (3), 229-233 (1995).

3. Kuraishi, Y., Yamaguchi, T., Miyamoto, T. Itch-scratch responses induced by opioids through central mu opioid receptors in mice. Journal of Biomedical Science. 7 (3), 248-252, (2000).

4. Shimada, S. G., LaMotte, R. H. Behavioral differentiation between itch and pain in mouse. Pain. 139 (3), 681-687, (2008).

5. Kittaka, H., Uchida, K., Fukuta, N., Tominaga, M. Lysophosphatidic acid-induced itch is mediated by signalling of LPA5 receptor, phospholipase D and TRPA1/TRPV1. The Journal of Physiology. 595 (8), 2681-2698, (2017).

6. Maekawa, T., Nojima, H., Kuraishi, Y. Itch-associated responses of afferent nerve innervating the murine skin: different effects of histamine and serotonin in ICR and ddY mice. The Japanese Journal of Pharmacology. 84 (4), 462-466 (2000).

7. Inagaki, N. et al. Scratching behavior in various strains of mice. Skin Pharmacology and Applied Skin Physiology. 14 (2), 87-96, (2001).

8. Inami, Y. et al. Topical surfactant-induced pruritus: involvement of histamine released from epidermal keratinocytes. Journal of Pharmacology and Experimental Therapeutics. 344 (2), 459-66, (2012).

9. Vos, B. P., Hans, G., Adriaensen, H. Behavioral assessment of facial pain in rats: face grooming patterns after painful and non-painful sensory disturbances in the territory of the rat's infraorbital nerve. Pain. 76 (1-2), 173-178 (1998).

10. Yeo, J. F., Ong, W. Y., Ling, S. F., Farooqui, A. A. Intracerebroventricular injection of phospholipases A2 inhibitors modulates allodynia after facial carrageenan injection in mice. Pain. 112 (1-2), 148-155, (2004).

11. Yeo, J. F., Ling, S. F., Tang, N., Ong, W. Y. Antinociceptive effect of CNS peroxynitrite scavenger in a mouse model of orofacial pain. Experimental Brain Research. 184 (3), 435-438, (2008).

12. Hashimoto, T., Ohata, H., Momose, K. Itch-scratch responses induced by lysophosphatidic acid in mice. Pharmacology. 72 (1), 51-56, (2004).

13. Kremer, A. E. et al. Lysophosphatidic acid is a potential mediator of cholestatic pruritus. Gastroenterology. 139 (3), 1008-1018, 1018 e1001, (2010).

14. Alemi, F. et al. The TGR5 receptor mediates bile acid-induced itch and analgesia. Journal of Clinical Investigation. 123 (4), 1513-1530, (2013).

15. Nieto-Posadas, A. et al. Lysophosphatidic acid directly activates TRPV1 through a C-terminal binding site. Nature Chemical Biology. 8 (1), 78-85, (2011).

16. Akiyama, T., Carstens, MI., Carstens, E. Differential itch- and pain-related behavioral responses and $\mu$-opoid modulation in mice. Acta Dermato-Venereologica. 90 (6), 575-581, (2010). 